begehungen an“, so Kriminaloberkommissar Rainer Both, Landespolizei Saarland. Man berate die Mediziner, wie sie sich besser gegen Einbrüche, aber auch gegen gewaltsame Übergriffe schützen können. „Dazu gehört, dass gefährliche Gegenstände nicht im Griff- und Sichtbereich der Patienten liegen sollten“, so Both. Ein Thema sei auch das Verlassen des Gebäudes. So könne man dafür sor- gen, dass der Weg bis zum Parkplatz gut ausgeleuchtet ist oder Absprachen treffen, dass der letzte Kollege die Praxis nie allein verlässt. Die Hauptrolle bei schweren Gewalttaten spiele die von Hoffmann erwähnte kalte Aggression. „Sie ist fokussiert, ruhig, strategisch geplant und die Emotionen sind ausgeschaltet", erläuterte Both. „Dabei funktioniert dann keine psychologische Deeskalati- on." Kalte Aggression baue sich meist in mehreren Stufen auf - von der Fokussierung über Drohungen bis hin zu Probehandlungen und Vorbereitungen wie der Beschaffung von Waffen. „Zeigt sich ein erhöhtes Risikopotenzial bei einem Patienten, dann ist ab einem bestimmten Punkt ein Netzwerk aus Polizei, Psychiater und Experten für Bedrohungsmanagement erforderlich." Andreas Kindel

\title{
Neuer Facharzt-EBM im Verzug
}

\section{Der Weiterentwicklungsprozess des neuen Facharzt-EBM zieht sich. Der Termin für das Inkrafttreten am 1. Januar 2016 kann nicht gehalten werden.}

$\mathrm{D}$ ie EBM-Reform geht in die Verlängerung: Nach dem neuen Zeitplan von KBV und GKV-Spitzenverband soll sie nun bis Ende März 2017 beendet sein. Drei Monate später, also ab 1. Juli 2017, könnten dann alle Vertragsärzte und -psychotherapeuten nach dem angepassten Regelwerk abrechnen, meldet die KBV. Das trifft vor allem die Fachärzte, denn der überarbeitete Hausarzt-EBM gilt seit Herbst 2013.
Ursprünglich sollte der neue FacharztEBM zum 1. Januar 2016 in Kraft treten. Bereits Anfang Juli dieses Jahres zeichnete sich jedoch $\mathrm{ab}$, dass dieser Termin nicht gehalten wird. Wegen der Komplexität hatten die Kassen laut einem KBVBericht damals vorgeschlagen, einzelne Aspekte der Reform stufenweise umzusetzen. Das hatte die Vertreterversammlung der KBV aber abgelehnt und - weil sie "ungewollte Honorarverwerfungen“ befürchtete - eine Reform in einem Zug gefordert, selbst wenn die Umsetzung dann erst 2017 erfolgen könne.

Ein Ziel der Reform ist es, die betriebswirtschaftliche Kalkulationsmethode weiterzuentwickeln und etwa die Praxiskosten besser zu erfassen. Gerade die Überprüfung und Anpassung des zugrunde liegenden Standardbewertungssystems (STABS) erweist sich laut KBV jedoch als aufwändig. Außerdem sollen wieder mehr Leistungen außerhalb der Pauschalen vergütet werden, um das Leistungsspektrum der Praxen sowie den veränderten Versorgungsbedarf der Versicherten besser im EBM abzubilden.

Rebekka Höhl

\section{"Es muss Spaß machen, bei Ihnen zu arbeiten!"}

Eine zeitgemäße Führungskultur ist eine wichtige Voraussetzung dafür, dass sich eine Praxis erfolgreich entwickelt. Das hängt unter anderem damit zusammen, dass in den meisten Arztpraxen die Ausgaben für Personal mit $40 \%$ oder mehr der größte Kostenblock sind. Aber auch die Zufriedenheit der Patienten ist gebunden an die Motivation des Praxisteams - seien dies MFA oder angestellte Ärzte. Den Zusammenhang zwischen diesen beiden Erfolgsfaktoren einer Praxis verdeutlichte der Fachanwalt für Arbeitsrecht Kai Höppner kürzlich beim Seminar „Personalführung und Mitarbeiterauswahl in der Praxis" in Braunschweig. Veranstalter waren die Deutsche Apotheker- und Ärztebank (apoBank) und das Institut für Wirtschaft und Praxis (IWP) in Kooperation mit der KV Niedersachsen. „Es muss Spaß machen, bei Ihnen zu arbeiten“, so der Appell Höppners. Das komme auch bei den Patienten an, es spreche sich herum, es mache sich bemerkbar bei der Suche nach neuen Mitarbeitern und „bestenfalls merken Sie das dann auch, wenn Sie auf Ihre Umsätze schauen", sagte der Arbeitsrechtler.

Zu einer zeitgemäßen Führungskultur gehört laut Höppner vor allem die kooperative Zusammenarbeit mit den Mitarbeitern. Es sei wichtig, MFA und angestellten Ärzten Freiräume zu lassen, damit sie ihre Arbeit selbstständig machen und sich dabei wenigstens teilweise selbst entfalten können. Zu koppeln sei diese Freiheit aber immer mit konkreten Zielen, auf die die Mitarbeiter im Laufe des Jahres zusteuern können und die dann in einem regelmäßig durchgeführten Gespräch zum Jahresende oder -beginn nachgehalten werden können. Solche "Kommunikationsrituale" wie Mitarbeitergespräche, so Höppner, seien auch wichtig, um fest- zustellen, wie zufrieden die Mitarbeiter mit ihrer Situation sind. Darüber hinaus seien regelmäßige Teamsitzungen in diesem Zusammenhang von Bedeutung. Dabei sind aktuelle Informationen zur Praxisentwicklung, etwa zur Erweiterung des Praxisspektrums, ebenso anzusprechen wie Fehler und Konflikte. Fehler sind immer als Chance zu sehen, aus ihnen zu lernen und damit zukünftige Fehler zu vermeiden. Nur so kann Offenheit erreicht werden und Fehler werden nicht unter den Teppich gekehrt.

Zu den zehn Geboten einer zeitgemäßen Mitarbeiterführung gehöre auch, dass die Praxisinhaber bereit seien, ein kritisches Feedback ernst zu nehmen und darauf zu reagieren, sagte Höppner. Nicht zuletzt motiviere die Wertschätzung guter Arbeit von Mitarbeitern dazu, sich auch in Zukunft ins Zeug zu legen. Hauke Gerlof 